\title{
High-stable basswood porous carbon anode activated by phosphoric acid for sodium ion battery
}

\author{
Zhipeng Xu, Ying Huang*, Ling Ding, Jiaxin Huang, Heng Gao, Yan Li, Tiehu Li \\ MOE Key Laboratory of Material Physics and Chemistry under Extraordinary \\ Conditions, School of Chemistry and Chemical Engineering, Northwestern \\ Polytechnical University, Xi'an 710072, PR China.
}

\section{Experimental Section}

\section{Synthesis of phosphorus-doped porous carbon}

Basswood is sawn into thin slices perpendicular to its growth direction. The thin slices are firstly washed with deionized water to remove dust and impurities from surface, and then vacuum dried at $80{ }^{\circ} \mathrm{C}$ for $20 \mathrm{~h}$. Then a certain amount of phosphoric acid is dissolved in deionized water and immersed in basswood slices according to mass ratio (the mass ratio of phosphoric acid to basswood is $2: 1,1: 1,1: 2$ ). All solution and mixture is dried at a high temperature of $120^{\circ} \mathrm{C}$ to remove water and vacuum dried at $80{ }^{\circ} \mathrm{C}$ for $24 \mathrm{~h}$. After that, the black gum is heat treatment at $500^{\circ} \mathrm{C}$ for $2 \mathrm{~h}$ under nitrogen atmosphere. Finally, obtained product is mixed with $2 \mathrm{M}$ hydrochloric acid and refluxed at $120^{\circ} \mathrm{C}$ for $2 \mathrm{~h}$ to obtain phosphorus-doped biomass carbon(PBC-21, PBC-11 and PBC-12). BC is obtained by carbonization without phosphoric acid (other

\footnotetext{
${ }^{*}$ Corresponding author.

E-mail address (Y. Huang): yingh@nwpu.edu.cn
} 
conditions are the same).

\section{Materials characterization}

The structure of samples was investigated by XRD (Rigaku, model 2500 system). TGA testing was performed on Model Q50 in air atmosphere with a temperature range of $30-500{ }^{\circ} \mathrm{C}$ and heating rate of $10^{\circ} \mathrm{C} \mathrm{min}^{-1}$. Alpha300R was used to obtain the Raman spectrum of sample. The microscopic morphology was observed by transmission electron microscopy (FETEM, FEI Talos F200X) and field emission scanning electron microscopy (FEI Verios G4). BET surface area and pore size distribution were measured by Micromeritics ASAP. XPS was carried out on Phoibos 100 spectrometer.

\section{Electrochemical measurements}

Porous carbon, PVDF and Super P were dissolved in N-methyl-2-pyrrolidone at a mass ratio of 7:2:1 and coated on a copper foil as working electrode. Mass loading of active material is $1.9-2.4 \mathrm{mg} \mathrm{cm}^{-2}$. CR2016 button battery was assembled in argonfilled glove box with sodium metal as counter electrode for electrochemical testing. Electrolyte for sodium-ion battery is $1.0 \mathrm{~mol} \mathrm{~L}^{-1} \mathrm{NaClO}_{4}$ in ethyl carbonate (EC) and diethyl carbonate (DEC) (1:1 by volume). Cyclic voltammetry and impedance of batteries were measured through electrochemical workstation. Meanwhile, the cycle performance and rate property were tested by multi-channel current static system Land. 

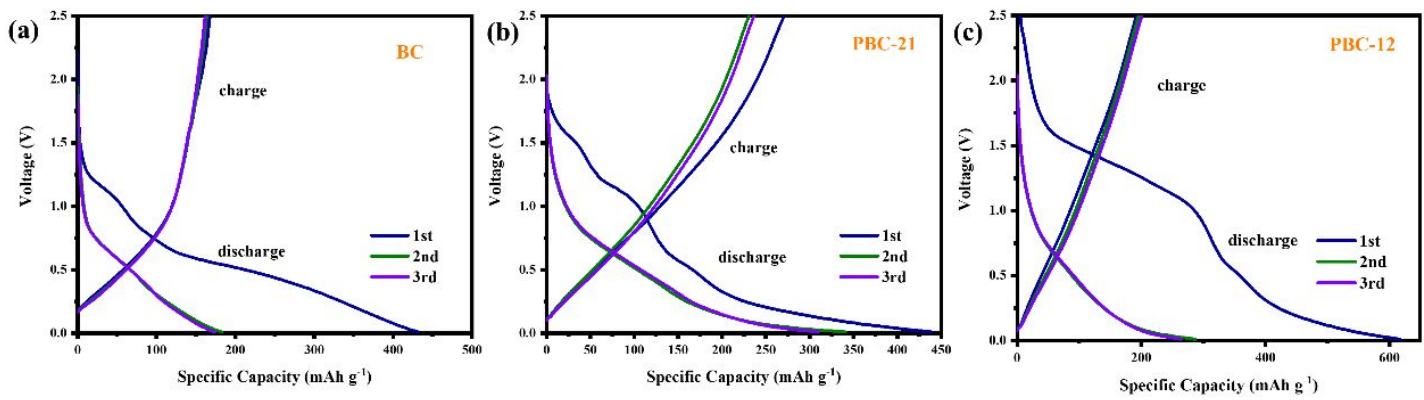

Fig. S1. The first three cycling charge-discharge curves of (a) BC, (b) PBC-21and (c) PBC-12.
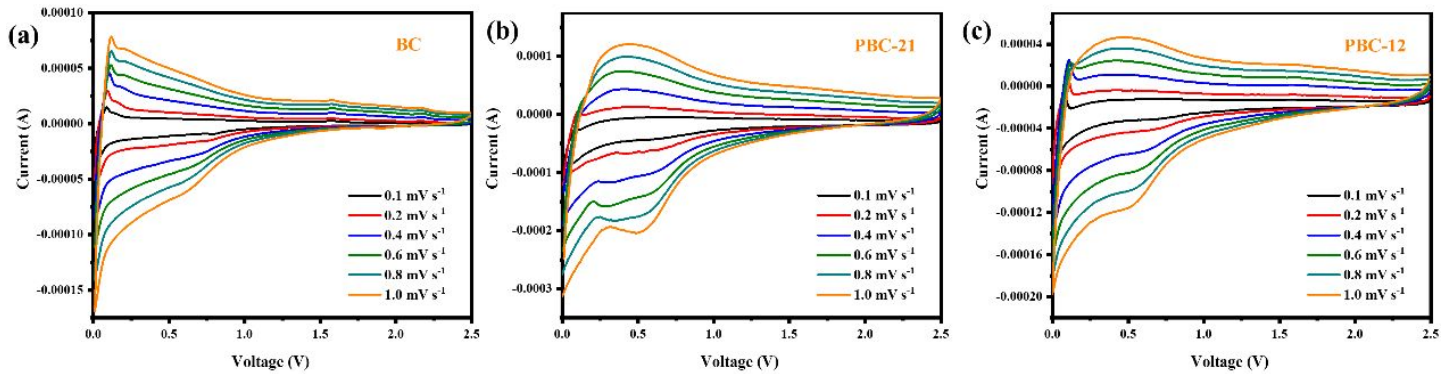

Fig. S2. The six CV curves of (a) BC (b) PBC-21 and (c) PBC-12 at $0.1-1 \mathrm{mV} / \mathrm{s}$ scan rates.

Table S1. Electrochemical impedance values of four electrode materials.

\begin{tabular}{ccc}
\hline electrode materials & $\mathrm{R}_{\mathrm{s}} / \Omega$ & $\mathrm{R}_{\mathrm{ct}} / \Omega$ \\
\hline BC before cycling & 5.16 & 63.88 \\
\hline PBC-21 before cycling & 4.56 & 49.71 \\
\hline PBC-11 before cycling & 3.99 & 35.78 \\
\hline PBC-12 before cycling & 6.51 & 66.76 \\
\hline BC after 500 cycles & 6.93 & 235.94 \\
\hline PBC-21 after 500 cycles & 6.10 & 171.81 \\
\hline PBC-11 after 500 cycles & 5.96 & 148.46 \\
\hline PBC-12 after 500 cycles & 7.56 & 177.08 \\
\hline
\end{tabular}


$D_{\mathrm{Na}}{ }^{+}$can be calculated by the following simplified formula $\mathrm{S} 1$. Where $\tau$ is the pulse duration, $m_{\mathrm{B}}$ represents the mass of the active material on the pole piece, $S$ is the area of the electrode, and $M_{\mathrm{B}}$ and $V_{\mathrm{m}}$ represent the molecular mass and molar volume of carbon, respectively. $\Delta E_{\mathrm{s}}$ and $\Delta E_{\tau}$ can be obtained from the GITT curve.

$$
D_{N a^{+}}=\frac{4}{\pi \tau}\left(\frac{m_{B} V_{m}}{M_{B} S}\right)^{2}\left(\frac{\Delta E_{s}}{\Delta E_{\tau}}\right)^{2}
$$

Table S2. Comparison of PBC-11 versus different biomass carbons.

\begin{tabular}{|c|c|c|c|c|c|}
\hline Samples & Biomass & $\begin{array}{l}\text { Current } \\
\text { density } \\
\left(\mathrm{mA} \mathrm{g}^{-1}\right)\end{array}$ & $\begin{array}{l}\text { Reversible } \\
\text { Capacity } \\
\left(\mathrm{mA} \mathrm{h} \mathrm{g}^{-1}\right)\end{array}$ & $\begin{array}{l}\text { Current } \\
\text { density } \\
\left(\mathrm{mA} \mathrm{g}^{-1}\right)\end{array}$ & $\begin{array}{l}\text { Cyclability } \\
\left(\mathrm{mA} \mathrm{h} \mathrm{g}^{-1}\right)\end{array}$ \\
\hline $\begin{array}{c}\text { PBC-11 } \\
\text { (this work) }\end{array}$ & Basswood & 100 & 326 & 2000 & $\begin{array}{c}165 \text { at } 3000 \text { th } \\
\text { cycle }\end{array}$ \\
\hline $\begin{array}{l}\mathrm{H}_{3} \mathrm{PO}_{4^{-}} \\
\text {activated } \\
\text { carbon[1] }\end{array}$ & $\begin{array}{l}\text { pomelo } \\
\text { peels }\end{array}$ & 50 & 287 & 200 & $\begin{array}{c}181 \text { at } 220 \text { th } \\
\text { cycle }\end{array}$ \\
\hline $\mathrm{CP}[2]$ & $\begin{array}{l}\text { cherry } \\
\text { petals }\end{array}$ & 20 & 298 & 500 & $\begin{array}{c}131 \text { at } 500 \text { th } \\
\text { cycle }\end{array}$ \\
\hline ACGL[3] & $\begin{array}{l}\text { ginkgo } \\
\text { leaves }\end{array}$ & 200 & 200 & 500 & $\begin{array}{c}125 \text { at } 500 \text { th } \\
\text { cycle }\end{array}$ \\
\hline $\mathrm{P} 1000[4]$ & $\begin{array}{c}\text { pistachio } \\
\text { shell }\end{array}$ & 10 & 225 & 40 & $\begin{array}{c}150 \text { at } 50 \text { th } \\
\text { cycle }\end{array}$ \\
\hline HPC-1-2[5] & $\begin{array}{l}\text { Peanut } \\
\text { skin }\end{array}$ & 100 & 226 & 500 & $\begin{array}{c}144 \text { at } 200 \text { th } \\
\text { cycle }\end{array}$ \\
\hline C-1000[6] & algae & 60 & 230 & 20 & 230 at 60 th \\
\hline
\end{tabular}




\begin{tabular}{|c|c|c|c|c|c|}
\hline & & & & & cycle \\
\hline $\mathrm{CC}[7]$ & corncob & 100 & 152 & 100 & $\begin{array}{c}139 \text { at } 100 \text { th } \\
\text { cycle }\end{array}$ \\
\hline $\mathrm{RFC}[7]$ & $\begin{array}{l}\text { Ramie } \\
\text { fiber }\end{array}$ & 100 & 133 & 100 & $\begin{array}{c}122 \text { at } 100 \text { th } \\
\text { cycle }\end{array}$ \\
\hline СРP [8] & pine pollen & 236 & 236 & 100 & $\begin{array}{c}201 \text { at } 100 \text { th } \\
\text { cycle }\end{array}$ \\
\hline
\end{tabular}

\section{References}

[1] K.L. Hong, Q. Long, R. Zeng, Z.Q. Yi, W. Zhang, D. Wang, W. Yin, C. Wu, Q.J. Fan, W.X. Zhang, Biomass derived hard carbon used as a high performance anode material for sodium ion batteries, J. Mater. Chem.A., 2 (2014) 12733-12738.

[2] Z. Zhu, F. Liang, Z. Zhou, X. Zeng, D. Wang, P. Dong, J. Zhao, S. Sun, Y. Zhang, X. Li, Expanded biomass-derived hard carbon with ultra-stable performance in sodiumion batteries, Journal of Materials Chemistry A, 6 (2018) 1513-1522.

[3] E. Hao, W. Liu, S. Liu, Y. Zhang, H. Wang, S. Chen, F. Cheng, S. Zhao, H. Yang, Rich sulfur doped porous carbon materials derived from ginkgo leaves for multiple electrochemical energy storage devices, J. Mater. Chem. A, 5 (2017) 2204-2214.

[4] K. Kim, D.G. Lim, C.W. Han, S. Osswald, V. Ortalan, J.P. Youngblood, V.G. Pol, Tailored Carbon Anodes Derived from Biomass for Sodium-Ion Storage, ACS. Sustain. Chem. Eng., 5 (2017) 8720-8728.

[5] H. Wang, W. Yu, J. Shi, N. Mao, S. Chen, W. Liu, Biomass derived hierarchical porous carbons as high-performance anodes for sodium-ion batteries, Electrochim. Acta, 188 (2015) 103-110. 
[6] X. Meng, P.E. Savage, D. Deng, Trash to Treasure: From Harmful Algal Blooms to High-Performance Electrodes for Sodium-Ion Batteries, Environ. Sci. Technol., 49 (2015) 12543-12550.

[7] J. Qiang, Z. Zhang, S. Yin, Z. Guo, S. Wang, C. Feng, Biomass carbon micro/nanostructures derived from ramie fibers and corncobs as anode materials for lithium-ion and sodium-ion batteries, Appl. Surf. Sci., 379 (2016) 73-82.

[8] Y. Zhang, X. Li, P. Dong, G. Wu, J. Xiao, X. Zeng, Y. Zhang, X. Sun, Honeycomblike Hard Carbon Derived from Pine Pollen as High-Performance Anode Material for Sodium-Ion Batteries, ACS Appl. Mater. Interfaces, 10 (2018) 42796-42803. 